\title{
A Retrospective Comparison between the PNST and other Paediatric Nutritional Screening Tools
}

\author{
Vesal Moeeni, Tony Walls and Andrew S. Day*
}

Department of Paediatrics, University of Otago/Christchurch, Christchurch School of Medicine, PO Box 4345, Christchurch 8041, New Zealand

\begin{abstract}
Background: Although it is widely acknowledged that hospitalized children are at greater risk of malnutrition, the available paediatric Nutritional Risk Screening (NRS) tools have not yet become universally used to identify those children at greater risk. Furthermore, the utility of one NRS tool over another remains unclear.

Materials and Methods: The utility of a recently developed tool, the Paediatric Nutritional Screening Tool (PNST), was evaluated using data previously collected in the assessment of three other NRS tools in 281 children from Iran and New Zealand. The sensitivity and specificity of each tool was then assessed based on the WHO criteria for malnutrition.

Results: The PNST recognized about half of the malnourished patients while the other three tools identified at least $85 \%$ of these children. The sensitivity of PNST for moderate (BMI-z $\leq 2)$ and severe malnutrition (BMI-z $<-3)$ was $37 \%$ and $46 \%$ respectively, while the sensitivity for other three NRS tools ranged from $82-100 \%$.

Conclusion: In this data set, the PNST tool did not perform as well as the three more established NRS tools. Further work is required to provide optimal tools for the identification of hospitalized children at risk of malnutrition.
\end{abstract}

Keywords: Malnutrition, Nutritional risk screening, paediatrics, children, hospital admission.

\section{INTRODUCTION}

Children who require hospitalization are at greater risk of malnutrition during their hospital stay [1]. Early detection and management of those children who are at risk of malnutrition, could prevent deterioration and adverse outcomes. Several screening tools have been developed for the assessment of the nutritional status of paediatric inpatients in recent years. These include the Screening Tool for the Assessment of Malnutrition in Paediatrics (STAMP) [2], Screening Tool for Risk On Nutritional status and Growth (STRONGkids) [3], Paediatric Yorkhill Malnutrition Score (PYMS) [4] and more recently, the Paediatric Nutritional Screening Tool (PNST) [5]. The four tools and their components are summarised in Supplementary Appendix 1. The goal of these screening tools is to provide a rapid evaluation to identify children at risk of deterioration of their nutritional state during their hospitalization. Identification may then enable to introduction of a specific nutritional intervention. Given the associations between malnutrition and nutritional risk with factors such as prolonged length of hospital stay, appropriate interventions may decrease morbidity and also reduce hospitalization-related costs.

While the PNST was developed in Australia, the other three tools were developed in European

*Address correspondence to this author at the Department of Paediatrics, University of Otago/Christchurch, PO Box 4345, Christchurch 8041, New Zealand; Tel: +64 3372 6760; E-mail: andrew.day@otago.ac.nz countries. The PNST is composed of four questions: these ask about recent unintentional weight loss, poor weight gain, loss of appetite or poor feeding and if the child is obviously under or overweight. The PNST was reported to be more sensitive, valid and simpler than other paediatric tools [5], but comparative analyses have not yet been performed.

This retrospective study aimed to compare the sensitivity and specificity of the PNST with that of the STRONGkids, STAMP and PYMS tools. This comparative analysis utilised previously collected data from a large group of children assessed upon their admission to hospital.

\section{METHODS}

\section{Study Design and Subjects}

The PNST tool scores were generated from a data set comprising 281 children aged between 12 months and 17 years (median age $5.3 \mathrm{yrs}$ ) at their admission to paediatric hospitals in Iran [6] or New Zealand [7]. As the study group was not normally distributed, median values were used for each demographic parameter. As previously described in detail, these two cohorts of children had been assessed with regards their nutritional status at the time of hospitalization. In addition, their risk of nutritional deterioration was previously assessed utilising the STRONGkids, PYMS and STAMP tools. 


\section{Classification of Malnutrition}

The World Health Organisation (WHO) classification was considered for recognition of malnutrition [8]. Children with weight-for-height (WFH) or height-for-age (HFA) <-3 z-score were considered severely malnourished. Moderate malnutrition was defined as WFH or HFA z-scores less than -2. BMI z-score was used for children older than 5 years $(<-3$ considered severe and $<-2$ moderate malnutrition). BMI percentile between $85-95 \%$ was considered as overweight and BMI percentile $>95 \%$ classified as obese [9]. Patients were classified using the PNST as either low, medium or high risk and as at risk or not at risk of malnutrition.

\section{Application of the PNST}

Overall, the questions included in PNST were similar and covered the same issues as the existing data available. The first question in the PNST (Has child unintentionally lost weight lately?) was considered similar to step 2 of the PYMS [10] and question 4 of the STRONGkids [3]. The second question (Has child had poor weight gain over the last few months?) was similar to question 4 of the STRONGkids. Question 3 (Has child been eating/feeding less in the last few weeks?) was the same as number 3 of the PYMS and STRONGkids, and step 2 of the STAMP tool $[11,12]$. The first part of question 4 (Is child obviously underweight/significantly overweight?) was considered equivalent to the first question of STRONGkids while the second part (being overweight) was also assessed according to previous data.

Therefore, each subject that was assessed in the two previous studies utilising the other three screening tools, was reassessed with the consideration of the similarity of the questions included in PNST. As well as assessing the population as a whole, each separate population (Iranian and NZ) was also reassessed separately, to evaluate the results in different populations.

\section{Statistical Analysis}

SPSS statistic 23 software (IBM Corporation, New York, USA) was used for statistical analysis. Chisquare was used for comparison of data between groups. Spearman's correlation was used to assess the relationships between anthropometric data and NRS tool results. The inter-rater agreement between pairs of tools were assessed by using Cohen's kappa coefficient test. The significance level was set at $<0.05$.

\section{RESULTS}

\section{The Characteristics of the Patients}

The patient group included 281 children altogether, with 119 from Iran and 162 from New Zealand (Table 1). The children were aged between 12 months and 17.16 years (median 5.3 years).

Table 1: Demographic characteristics of the 281 hospitalised children included in the current analysis. Children were recruited at hospitalization in either Iran or New Zealand (NZ). HFA = height for age, WFA = weight for age

\begin{tabular}{|c|c|}
\hline Characteristics & \\
\hline \hline Country (number, percentage) & $119(42.3)$ \\
Iran & $162(57.7)$ \\
\hline Age (median, range) & \\
Iranian & $5.9(1-17.16)$ \\
NZ & $5.1(1-15.8)$ \\
WFA z-score (median) & -0.76 \\
Iranian & 0.32 \\
NZ & -0.029 \\
HFA z-score (median) & -0.29 \\
Iranian & \\
NZ & \\
Length of stay (Median and & $2.08(1.00-25.63)$ \\
range in days) & $2.16(1.00-13.00)$ \\
Iran & $2.00(1.00-24.63)$ \\
NZ &
\end{tabular}

\section{Identification of Malnutrition by the Four Tools}

The number of malnourished patients was determined for each of the four tools (Table 2). The PNST was able to recognize $51 \%$ of malnourished patients ( $43 \%$ of NZ \& $54 \%$ of Iranian children), while each of the other three tools was able to recognize more than $85 \%$ of the children.

\section{Relationships between $z$ Scores and Score Performance}

The sensitivity, specificity, positive and negative predictive values of each tool for various z-scores were assessed (Table 3). The sensitivity of PNST in recognizing severely malnourished patients based on HFAZ or BMIZ scores, was only $37 \%$ compared to $100 \%$ for the other three tools. The sensitivity of PNST 
Table 2: Comparison between the paediatric NRS tools in regards the recognition of malnutrition. The number of children recognized to be malnourished by the WHO classification (as the gold standard) was evaluated for each of the four nutritional risk screening tools

\begin{tabular}{|c|c|c|c|c|}
\hline $\begin{array}{c}\text { Malnourished } \\
\text { Based on WHO criteria }\end{array}$ & $\begin{array}{c}\text { Recognized by } \\
\text { PNST }\end{array}$ & $\begin{array}{l}\text { Recognized by } \\
\text { STRONGkids }\end{array}$ & $\begin{array}{l}\text { Recognized by } \\
\text { STAMP }\end{array}$ & $\begin{array}{c}\text { Recognized by } \\
\text { PYMS }\end{array}$ \\
\hline $\begin{array}{c}\text { Total } 47 \\
\text { NZ } 14 \\
\text { Iran } 33\end{array}$ & $\begin{array}{c}24(51 \%) \\
6(43 \%) \\
18(54 \%)\end{array}$ & $\begin{array}{c}41(87 \%) \\
14(100 \%) \\
27(82 \%)\end{array}$ & $\begin{array}{c}42(89 \%) \\
9(64 \%) \\
20(60 \%)\end{array}$ & $\begin{array}{l}40(85 \%) \\
11(78 \%) \\
29(96 \%)\end{array}$ \\
\hline
\end{tabular}

Table 3: Sensitivity, Specificity, and Predictive Values for the PNST score cut-offs and the STRONGkids score cut-offs in the identification of malnourished children. Sens $=$ sensitivity, Spec $=$ specificity, PPV $=$ positive predictive value, NPV = negative predictive value, HFA = height for age, WFA = weight for age, BMI - body mass index

\begin{tabular}{|c|c|c|c|c|c|c|c|c|c|c|c|c|c|c|c|c|c|}
\hline \multirow{3}{*}{ Measures } & \multicolumn{5}{|c|}{ Risk identified by PNST } & \multicolumn{4}{|c|}{ Risk by STRONGkids } & \multicolumn{4}{|c|}{ Risk by STAMP } & \multicolumn{4}{|c|}{ Risk by PYMS } \\
\hline & No. (\%) & Sensitivity & Specificity & PPV & NPV & Sen. & Sp. & PPV & PPV & Sen. & Sp. & PPV & NPV & Sen. & Sp. & PPV & NPV \\
\hline & $75(27)$ & NA & NA & NA & NA & NA & NA & NA & NA & NA & NA & NA & NA & NA & NA & NA & NA \\
\hline \multicolumn{18}{|c|}{ (> 2 affirmative Responses) WFAZ } \\
\hline$\leq-2$ & $32(11)$ & 50 & 82 & 25 & 95 & 99 & 43 & 17 & 99 & 100 & 60 & 16 & 100 & 89 & 67 & 21 & 98 \\
\hline$\leq-3$ & $7(2)$ & 57 & 79 & 5 & 99 & 100 & 62 & 4 & 100 & 100 & 59 & 35 & 100 & 100 & 66 & 47 & 100 \\
\hline \multicolumn{18}{|l|}{ HFAZ } \\
\hline$\leq-2$ & $16(6)$ & 37 & 79 & 8 & 96 & 87 & 60 & 12 & 99 & 94 & 59 & 7 & 99 & 68 & 64 & 7 & 98 \\
\hline$\leq-3$ & $8(3)$ & 37 & 79 & 4 & 98 & 100 & 70 & 6 & 100 & 100 & 59 & 4 & 100 & 100 & 66 & 5 & 100 \\
\hline \multicolumn{18}{|l|}{ BMIZ } \\
\hline$\leq-2$ & $28(10)$ & 46 & 80 & 17 & 94 & 89 & 61 & 20 & 97 & 82 & 60 & 12 & 98 & 96 & 68 & 18 & 99 \\
\hline$\leq-3$ & $8(3)$ & 37 & 79 & 4 & 98 & 100 & 70 & 6 & 100 & 100 & 61 & 4 & 100 & 100 & 66 & 5 & 100 \\
\hline
\end{tabular}

was $37 \%$ (HFAZ) and $46 \%$ (BMIZ) for moderate malnutrition versus $68-100 \%$ for the other 3 tools.

The correlations between WFA, HFA and BMI zscores and all four tools were determined (Table 4). None of the tools had a good correlation with the zscores.

\section{The Agreements between Different NRS Tools}

The inter-rater agreement between each pair of tools was assessed and kappa coefficients were compared (Table 5). The highest agreements were between STRONGkids and STAMP, and between STRONGkids and PYMS, while the PNST tool had the

Table 4: Correlation between the nutrition risk identified by the PNST and the nutrition risk identified by three other tools and nutrition status by $z$ scores. HFA = height for age, WFA = weight for age, BMI - body mass index

\begin{tabular}{|c|c|c|c|}
\hline Measure & $\begin{array}{c}\text { Correlation } \\
\text { PNST }\end{array}$ & $\begin{array}{c}\text { Correlation } \\
\text { STRONGkids }\end{array}$ & $\begin{array}{c}\text { Correlation } \\
\text { STAMP }\end{array}$ \\
\hline \hline WFAZ & -0.17 & -0.08 & -0.27 \\
$\leq-2$ & 0.17 & 0.31 & -0.32 \\
S-3 & & & -0.65 \\
\hline HFAZ & -0.13 & -0.19 & -0.49 \\
S-2 & -0.04 & -0.57 & -0.30 \\
\hline$\leq-3$ & & & -0.28 \\
\hline BMIZ & 0.09 & -0.07 & -0.15 \\
\hline-2 & 0.22 & 0.18 & -0.13 \\
\hline
\end{tabular}


Table 5: Kappa value and inter-rater agreement between the four different tools

\begin{tabular}{|c|c|c|c|c|}
\hline & STRONGkids & STAMP & PYMS & PNST \\
\hline \hline STRONGkids & 1 & 0.57 & 0.51 & 0.34 \\
\hline STAMP & 0.57 & 1 & 0.36 & 0.2 \\
\hline PYMS & 0.51 & 0.36 & 0.46 \\
\hline PNST & 0.34 & 0.2 & 0.46 & 1 \\
\hline
\end{tabular}

lowest agreements with those two tools and a moderate agreement with the PYMS tool.

\section{DISCUSSION}

This study has demonstrated that the PNST tool was able to recognize approximately half as many of the malnourished children as the other three tools in this historical data set. In addition, the sensitivity of this tool was also substantially less than that of the other tools $(37 \%$ versus $68-100 \%)$.

An earlier study conducted in Belgium evaluated the STRONGkids tool and demonstrated similar sensitivity $(69-71.9 \%)$ for this tool [13]. The original study describing the development and validation of the PYMS tool, conducted on 247 paediatric inpatients, also determined high sensitivity for this tool $(85 \%)$ and compared it with the STAMP tool (81\%) [14]. In contrast, a more recent report that included assessment of STRONGkids, PYMS and STAMP in 2567 inpatients in 12 European countries indicated lower utility for these three tools [15]. With regards to the utility of these various tools, Hartman et al. [16] has commented that a standardized approach for nutritional screening for paediatric inpatients has not yet been achieved.

The PNST is designed to be simple and rapid, without a requirement for collection of anthropometric measurements. Similarly, the STRONGkids tool does not include collection of weight and height data, whereas STAMP and PYMS do require this data [2-4]. The questions included in the PNST essentially cover the same aspects as included in the previous tools, especially the STRONGkids tool. This allows for reliable retrospective application of the PNST tool in these two cohorts of children. The predominant difference between PNST and STRONGkids is that PNST excludes information about underlying chronic disease. The lack of consideration of the impact of underlying disease could contribute to the differences in the observed sensitivities between these tools and could explain why PNST has lower identification rate of malnutrition.

Due to increasing prevalence of overweight/obesity in children in NZ and many other countries, the consideration of this as a risk factor for malnutrition is advisable. Although the PNST asks about the existence of overweight/obesity, the authors have noted poor sensitivity and specificity in identification of these patients [5]. The current study also showed that there was not a good correlation between z-scores and any of the NRS tools as shown previously [5].

A limitation of this report is that the data was collected retrospectively meaning that the PNST was not applied directly to each patient during their hospitalization but was derived from the previously collected data. This may diminish the accuracy of the conclusions. However, the similarity of the questions included in PNST and the large comprehensive nature of the data set should mitigate against this.

In conclusion, this analysis of previously-collected data illustrates that the PNST has low sensitivity for the recognition of malnutrition in paediatric inpatients when compared to the other three validated NRS tools. Further consideration of the utility of NRS tools in children admitted to hospital is required.

\section{REFERENCES}

[1] Malnutrition. The Starvelings [Internet] [updated 2008 Jan 24 cited 2018 Mar 6. Available from: http://www.economist.com/ world/international/displaystory.cfm?story_id=10566634.

[2] McCarthy H, Dixon M, Crabtree I, Eaton-Evans MJ, McNulty $\mathrm{H}$. The development and evaluation of the Screening Tool for the Assessment of Malnutrition in Paediatrics (STAMP) for use by healthcare staff. J Hum Nutr Diet 2012; 25: 311-8. https://doi.org/10.1111/j.1365-277X.2012.01234.X

[3] Hulst JM, Zwart H, Hop WC, Joosten KFM. Dutch national survey to test the STRONGkids nutritional risk screening tool in hospitalized children. Clin Nutr 2010; 29: 106-11. https://doi.org/10.1016/j.clnu.2009.07.006

[4] Nutrition Tool Steering Group Women and Children's Directorate. Paediatric Yorkhill Malnutrition Score (PYMS) information and user's guide. Glasgow: NHS Greater Glasgow and Clyde 2009 
[5] White M, Lawson K, Ramsey R, et al. A simple nutritional screening tool for pediatric inpatients. J Parenter Enter Nutr 2016; 40: 392-8. https://doi.org/10.1177/0148607114544321

[6] Moeeni V, Walls T, Day AS. Assessment of nutritional status and nutritional risk in hospitalised Iranian children. Acta Paediatr 2012; 101: e446-51. https://doi.org/10.1111/j.1651-2227.2012.02789.x

[7] Moeeni V, Walls T, Day AS. Nutritional status and nutrition risk screening in hospitalized children in New Zealand. Acta Paediatr 2013; 102: e419-23. https://doi.org/10.1111/apa.12299

[8] World Health Organisation. The WHO Child Growth Standards [Internet] 2007 [cited 2018 Feb 5]. Available from: http://www.who.int/childgrowth/en/

[9] Center for Disease Control and prevention. Obesity and Overweight for Professionals: Childhood: Basics [Internet] 2012 [updated 2012 Apr 27; cited 2018 Feb 14. Available from: http://www.cdc.gov/obesity/childhood/basics.html.

[10] Gerasimidis K, Macleoda I, Macleana A, et al. Performance of the novel Paediatric Yorkhill Malnutrition Score (PYMS) in hospital practice. Clin Nutr 2011; 30: 430-5. https://doi.org/10.1016/j.clnu.2011.01.015
[11] McCarthy $H$, McNulty $H$, Dixon $M$, Eaton-Evans $M J$. Screening for nutrition risk in children: the validation of a new tool. J Hum Nutr Diet 2008; 21: 395-6. https://doi.org/10.1111/j.1365-277X.2008.00881 31.x

[12] McCarthy H. STAMP instruction [Internet] 2008 [updated 2010 June]. [cited 2018 Feb 5]. Available from: http://www.stampscreeningtool.org/help/index.html

[13] Huysentruyt KM, Alliet P, Muyshont L, et al. The STRONGkids nutritional screening tool in hospitalized children: A validation study. Nutrition 2013; 29: 1356-61. https://doi.org/10.1016/j.nut.2013.05.008

[14] Gerasimidis K, Keane O, Macleod I, Flynn DM, Wright CM. A four-stage evaluation of the Paediatric Yorkhill Malnutrition Score in a tertiary paediatric hospital and a district general hospital. Br J Nutr 2010; 104: 751-6. https://doi.org/10.1017/S0007114510001121

[15] Chourdakis M, Hecht C, Gerasimidis K, et al. Malnutrition risk in hospitalized children: use of 3 screening tools in a large European population. Am J Clin Nutr 2016; 103: 1301-10. https://doi.org/10.3945/ajcn.115.110700

[16] Hartman C, Shamir R, Hecht C, B K. Malnutrition screening tools for hospitalized children. Curr Opin Clin Nutr Metab Care 2012; 15: 303-9. https://doi.org/10.1097/MCO.0b013e328352dcd4

https://doi.org/10.6000/1929-4247.2018.07.03.1

(c) 2018 Moeeni et al.; Licensee Lifescience Global.

This is an open access article licensed under the terms of the Creative Commons Attribution Non-Commercial License (http://creativecommons.org/licenses/by-nc/3.0/) which permits unrestricted, non-commercial use, distribution and reproduction in any medium, provided the work is properly cited. 